\title{
Anesthesia in a child with adrenoleukodystrophy
}

\author{
Hun-Ju Yang, Ji-Eun Kim, Tae-Yun Sung, Choon-Kyu Cho, and Po-Soon Kang \\ Department of Anesthesiology and Pain Medicine, Konyang University Hospital, Konyang University College of Medicine, Daejeon, \\ Korea
}

$\mathrm{X}$-linked adrenoleukodystrophy (ALD) is rare genetic disorder, and children with ALD are at an increased risk of anesthetic mortality and morbidity [1]. Therefore, they require an individual anesthetic protocol based on their clinical condition. Herein we report the case of a male with childhood-onset X-linked ALD who required general anesthesia for a dental operation.

An 11-year-old male (height $143 \mathrm{~cm}$, weight $30 \mathrm{~kg}$ ) was scheduled for scaling and multiple deciduous tooth extractions under general anesthesia due to his failure to cooperate willingly.

He had been diagnosed with childhood-onset X-linked ALD 3 years previously. Other history included an episode of status epilepticus, adrenal insufficiency, aspiration pneumonia, pressure sores and blindness. He was bed-ridden and maintained on a positive-pressure home ventilator via tracheostomy. Physical examination revealed spastic tetraparesis, unresponsiveness to the environment, and cushingoid facies due to chronic steroid supplementation.

Preoperative medications including topiramate, divalproex, prednisolone were continued until the morning of the operation. On arrival at the operating room, standard monitoring commenced. For anesthetic induction, $75 \mathrm{mg}$ of pentothal sodium were injected. Anesthesia was maintained using $\mathrm{N}_{2} \mathrm{O}$ at $2 \mathrm{~L} / \mathrm{min}, \mathrm{O}_{2}$ at $2 \mathrm{~L} / \mathrm{min}$, and sevoflurane. About $5 \mathrm{~min}$ after the start of maintenance inhalational anesthesia, the surgeon was unable to open the patient's mouth so rocuronium $10 \mathrm{mg}$ was injected, after which the mouth could be opened and the surgery commenced. The operation lasted $30 \mathrm{~min}$ and was uneventful. Residual neuromuscular blockade was reversed with pyridostigmine $10 \mathrm{mg}$ and glycopyrrolate $0.2 \mathrm{mg}$, and $15 \mathrm{~min}$ after administration of reversal agents, we placed the patient's home ventilator in pressure-support ventilation (PSV) mode, as it was preoperatively. The patient was observed closely for over $1 \mathrm{~h}$ by an anesthesiologist and a pediatrician in the post-anesthesia care unit (PACU). The home ventilator with PSV mode was well tolerated, so we then transferred the patient to the general ward under monitoring with pulse oximetry.

$\mathrm{X}$-linked ALD is a progressive neurodegenerative disorder characterized by demyelination of cerebral white matter, axonopathy of the spinal cord, and reduced adrenal response to adrenocorticotropic hormone. The onset age of the condition is between 3 and 10 years, and the patients initially present with behavioral problems and deficits in memory and language. As the disease progresses, vision loss, auditory impairment, hemiparesis and spastic tetraparesis may occur. In the later stages, seizures begin and the patient becomes bedridden, blind, and unable to interact with the environment. Death usually ensues within 2 to 4 years after the onset of symptoms [1].

There are several considerations in anesthetic management for these patients. The anesthetic plan should be formulated based on each individual patient's condition according to their disease progression. In our case, the anesthetic plan focused on the prevention of seizures and early return to the preoperative condition without any anesthesia-related complications.

In an ALD patient with seizure disorders, anticonvulsants should be continued throughout the perioperative period, including the day of surgery, and the anesthesiologist should consider the altered pharmacokinetics of anesthetics due to hepatic microsomal enzyme induction secondary to chronic antiseizure

Corresponding author: Tae-Yun Sung, M.D., Department of Anesthesiology and Pain Medicine, Konyang University Hospital, Konyang University College of Medicine, 158, Gwangeodong-ro, Seo-gu, Daejeon 302-718, Korea. Tel: 82-42-600-9316, Fax: 82-42-545-2132, E-mail: unt1231@naver.com

(c) This is an open-access article distributed under the terms of the Creative Commons Attribution Non-Commercial License (http:// creativecommons.org/licenses/by-nc/3.0/), which permits unrestricted non-commercial use, distribution, and reproduction in any medium, provided the original work is properly cited. 
therapy. Also, anesthetics with epileptogenic potential, such as enflurane, ketamine, atracurium, cisatracurium, and meperidine, should be avoided [2].

Sedative premedication should also be avoided because of the risk of airway compromise due to hypotonic pharyngeal muscles. By contrast, $\mathrm{H} 2$-antagonists and glycopyrrolate are recommended to reduce the risk of aspiration pneumonia and oral secretions.

As in the case reported by Hamdiye et al. [3] and ours, some patients with ALD may have limited mouth opening, necessitating fiber-optic intubation with muscle relaxants or tracheostomy. Moreover, most patients with ALD have an increased risk of gastroesophageal reflux (GER) due to gastric dismotility, requiring modified rapid sequence induction with a non-depolarizing muscle relaxant with cricoid pressure [4]. In the present case, although our patient had limited mouth opening, he already had a tracheostomy, so we administered only a small dose of pentothal sodium for anesthesia induction. For anesthesia induction and maintenance, thiopental, propofol, $\mathrm{N}_{2} \mathrm{O}$, sevoflurane, isoflurane have been previously used successfully in these patients $[3,4]$.

The use of muscle relaxants may differ according to the type of surgery, and vecuronium, rocuronium, and mivacurium have been used without complications $[3,4]$. However, considering the susceptibility to pulmonary complications and early recovery of respiratory function, for surgeries that do not require muscle relaxation, application of cricoids pressure until intubation and avoidance of muscle relaxants are desirable. If surgical relaxation is needed, minimal doses of short-acting muscle relaxants are advisable. Succinylcholine is not recommended due to the risks of hyperkalemia in patients with neuromuscular disease [4].

Intraoperative monitoring parameters, such as train-offour and bispectral index (BIS), must be interpreted cautiously. Neuromuscular monitoring by peripheral nerve stimulation could result in overdosing of competitive neuromuscular blocking agents because of the refractory response to neuromuscular blockade in areas of hemiplegia or nerve damage. Also, the BIS values can decrease or increase regardless of the level of sedation in critically ill children according to their conditions, such as seizures, cerebral ischemia, and hypoglycemia [5].

Estimation of recovery from anesthesia and the decision to discharge from PACU can be difficult because during $\sim 2 \mathrm{~h}$ of observation after the surgery was completed, the patient in our case did not show any signs of recovery, with the exception of intermittent spontaneous respiration. Patients with later-stage ALD are often in a nearly vegetative state and may have preexisting respiratory complications such as airway obstruction, hypoventilation, and hypoxemia. Therefore, extended observation in the PACU is necessary.

In conclusion, although childhood-onset X-linked ALD is eventually fatal and has no definitive treatment, meticulous preoperative evaluation based on associated conditions is essential for anesthetic management. Prevention of seizures and GER, altered pharmacokinetics of anesthetics, potentially difficult intubation, and delayed recovery should be considered when designing the individual protocol of each patient.

\section{References}

1. Engelen M, Kemp S, de Visser M, van Geel BM, Wander RJ, Aubourg P, et al. X-linked adrenoleukodystrophy (X-ALD): clinical presentation and guidelines for diagnosis, follow-up and management. Orphanet J Rare Dis 2012; 7: 51.

2. Butterworth JF, Mackey DC, Wasnick JD. Anesthesia for Ptients with Neurologic \& Psychiatric Diseases. In: Morgan and Mikhail's Clinical Anesthesiology. 5th ed. New York, McGraw-Hill. 2013, pp 616-8.

3. Hamdiye CT, Yavuz G, Kamil T, Mine S. Anesthesia management of a child with adrenoleucodystrophy. Paediatr Anaesth 2006; 16: $221-2$.

4. Dobson G, Lyons J. Anaesthesia for life-limited child with adrenoleucodystrophy. Eur J Anaesthesiol 2004; 21: 78-9.

5. Lamas A, Lopez-Herce J. Monitoring sedation in the critically ill child. Anaesthesia 2010; 65: 516-24. 